\title{
Nukleinsäure-Amplifikationstests für HIV, HBV und HCV bei Gewebespendern: Sinnvoll oder überflüssig?
}

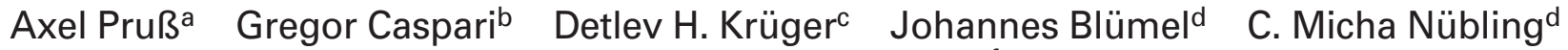 \\ Ernst-Markus Quenzele Ulrich Kalus ${ }^{\mathrm{a}}$ Wolfram Gerlich ${ }^{\mathrm{f}}$ Lutz Gürtler ${ }^{\mathrm{g}}$ \\ ${ }^{a}$ Gewebebank, Institut für Transfusionsmedizin, Charité - Universitätsmedizin Berlin, Campus Mitte, Berlin, \\ ${ }^{b}$ Zentrale Einrichtung Medizinaluntersuchungsamt und Krankenhaushygiene, Universitätsklinikum Schleswig-Holstein, Campus Kiel, \\ c Institut für Virologie (Helmut-Ruska-Haus), Charité - Universitätsmedizin Berlin, Campus Mitte, Berlin, \\ ${ }^{\mathrm{d}}$ Abteilung Virologie, Paul-Ehrlich-Institut, Langen, \\ e Institut für Transfusionsmedizin, Helios-Klinikum Schwerin, \\ ${ }^{f}$ Institut für Medizinische Virologie, Justus-Liebig-Universität Gießen, \\ gInstitut für Medizinische Virologie, Universitätsklinikum Frankfurt, Frankfurt/M., Deutschland
}

Schlüsselwörter

Allograft · Gewebebank · Virussicherheit · NAT · HIV . $\mathrm{HCV} \cdot \mathrm{HBV}$

\section{Zusammenfassung}

Mit der Umsetzung der EU-Richtlinien 2004/23/EG und 2006/17/EG im Gewebegesetz sowie den begleitenden Verordnungen (Arzneimittel- und Wirkstoffherstellungsverordnung, Transplantationsgesetz-Gewebeverordnung) wurden die grundlegenden Anforderungen an die Virussicherheit der Gewebespenden allgemein definiert. Während infektionsserologische Testungen (Anti-HIV 1/2, Anti-HCV Hepatitis-B-Oberflächenantigen, Anti-Hepatitis-B-Core-Antigen, Treponema-pallidum-Hämagglutinationsassay) vorgeschrieben sind, wird der Nukleinsäure-Nachweis für HIV, HBV und HCV nicht explizit gefordert. Anhand in der Literatur berichteter Virusübertragungen, gewebespezifischer Besonderheiten sowie Herstellungs- und gegebenenfalls Inaktivierungsverfahren wird eine Bewertung des Stellenwertes des HIV/HCV/HBV-Nachweises mittels Nukleinsäure-Amplifikationstechniken (NAT) bei Spendern unterschiedlicher Gewebe vorgenommen und mit den Erfahrungen des Blutspendewesens verglichen. Muskuloskelettale Gewebe besitzen infolge des zumeist hohen Blutgehalts der Gewebe, des umfangreichen Entnahmespektrums, der bisher beschriebenen Übertragungen, der unterschiedlichen Herstellungsverfahren sowie der hohen Spender-EmpfängerRatio ein signifikantes Risiko, HIV/HCV/HBV zu übertragen. Daher sollte bei Spendern muskuloskelettaler Gewebe, die keinem effektiven Virusinaktivierungsverfahren unterzogen werden, eine HIVHBV- und HCV-NAT-Testung erfolgen. Kardiovaskuläres Gewebe hat bei Durchführung der serologischen Testung ein sehr geringes Restrisiko der HIV/HCV/HBV-Übertragung. Aufgrund der fehlenden Möglichkeit einer effektiven Virusinaktivierung (Erhalt der Gewebemorphologie) und der Spender-Empfänger-Ratio von bis zu 1:10 sollte die HIV-, HCV- und HBV-NAT jedoch als zusätzliche Sicherheitsmaßnahme erfolgen. Augenhornhäute besitzen aus physiologisch-morphologischer sowie epidemiologischer Sicht das geringste HIV/HCV/HBV-Übertragungsrisiko, jedoch sollte die HCV-NAT durchgeführt werden. Eine Quarantänelagerung der Gewebe eines Spenders kann bei negativem Ergebnis der HIV/HCV/HBV-NAT grundsätzlich entfallen. Aufgrund der vielfältigen Synergieeffekte mit der Transfusionsmedizin bietet es sich für Gewebebanken an, die Testung der infektionsserologischen bzw. molekularbiologischen Laborparameter in Kooperation mit Blutspendediensten durchzuführen.
Key Words

Allograft · Tissue bank · Virus safety · HIV · HBV · HCV .

Nucleic acid testing

\section{Summary}

Nucleic Acid Amplification Tests for HIV, HBV, und HCV in Tissue

Donors: Useful or Dispensable?

With the conversion of the EU-Guidelines 2004/23/EC and 2006/17/EC in the tissue law as well as the accompanying regulations (AMWHV, TPG-GewV), the fundamental requirements were defined for the virus safety of tissue donations. While serologic testing (anti-HIV 1/2, anti-HCV, hepatitis B surface antigen, anti-hepatitis B core antigen, Treponema pallidum hemagglutination assay) is compulsory, nucleic acid analysis for HIV, HBV and HCV is not explicitly required. On the basis of virus transmissions, tissue-specific characteristics as well as manufacture and, where applicable, inactivating procedures reported in the literature, an evaluation was made of the significance of HIV/HCV/HBV detection by nucleic acid amplification techniques (NAT) in donors of various tissues and compared with the experiences with blood donations. Musculoskeletal tissues possess a significant risk of transmitting HIV/HCV/ HBV as a result of the mostly high blood content in tissues, the comprehensive extraction spectrum, the transmissions described to date, the different manufacturing processes as well as the high donor-recipient ratio. Therefore, with donated musculoskeletal tissues that do not undergo an effective virus-inactivating procedure, HIV-, HCV- und HBV-NAT testing should be performed. Serological testing assures that cardiovascular tissue has a very small residual risk of HIV/HCV/HBV transmission. Due to the impossibility of an effective virus inactivation (preservation of the tissue morphology) and a donor-recipient ratio of up to 1:10, HIV-, HCV- and HBV-NAT should, however, be performed as an additional safety precaution. Corneas possess the smallest HIV/HBV/HCV transmission risk from a physiological, morphological as well as an epidemiological viewpoint; however, HCV-NAT should be performed. In principle, quarantine storage of the tissues from a donor can be omitted in cases of negative HIV/HCV/HBV-NAT results. Due to the multifaceted synergetic effects with transfusion medicine, an obvious choice for tissue banks is the implementation of infectious serology and/or molecular-biological laboratory parameters in cooperation with blood bank services.

\section{KARGER}

Fax +497614520714

Information@Karger.de

www.karger.com
() 2008 S. Karger GmbH, Freiburg

Accessible online at:

www.karger.com/tmh
PD Dr. med. Axel Pruß

Gewebebank, Institut für Transfusionsmedizin

Charité - Universitätsmedizin Berlin, Campus Mitte

Charitéplatz 1, 10117 Berlin, Deutschland

Tel. +49 30 450525-161, Fax -976

axel.pruss@charite.de 


\section{Einleitung}

Trotz verstärkter Bemühungen, Alternativen zur allogenen Transplantation von muskuloskelettalen und kardiovaskulären Geweben sowie Augenhornhaut zu entwickeln, sind humane Gewebe für die Behandlung ausgedehnter Gewebedefekte weiterhin unverzichtbar.

Durch Auswahl der Spender (Anamnese, körperliche Untersuchung, Laboruntersuchungen) und sorgfältige Führung der Gewebeeinrichtungen werden die Risiken der Infektionsübertragung zum Schutz der Empfänger minimiert.

Derzeit wird die Frage diskutiert, in welchem Maße zusätzliche Untersuchungen des Gewebespenders auf die Genome von HIV, HCV und HBV mittels Nukleinsäure-Amplifikationstechniken (NAT) die Sicherheit der Gewebeübertragung weiter verbessern können und ob dieser Sicherheitsgewinn die Nachteile der zusätzlichen Untersuchungen (Inhibitionseffekte durch postmortale Blutproben und damit keine Freigabe der Gewebe, hohe Kosten) rechtfertigen kann.

$\mathrm{Zu}$ einer analogen Thematik, der Untersuchung von Blut- und Plasmaspenden für die Herstellung von Blutprodukten und Plasmaderivaten, gibt es mit der Hämotherapie-Richtlinie der Bundesärztekammer (BÄK) ein verbindliches Regelwerk, das durch Stufenpläne des Paul-Ehrlich-Instituts (PEI) und Voten des Arbeitskreises Blut ergänzt und weiterentwickelt wird. Das Standardverfahren der Testung auf HCV- und HIV-Infektionen ist der Antikörpertest, der positiv wird, wenn das Immunsystem des Spenders mit dem jeweiligen Virus reagiert. Bei HBV wird auf einen Bestandteil der Virushülle (HBVOberflächenantigen (HBsAg)) getestet. Frisch HCV- und HIV-infizierte Spender können infektiös sein, bevor der Antikörpertest positiv wird (serologische Latenzperiode bzw. diagnostisches Fenster). Für HBV-infizierte Spender trifft dies für eine kurze Phase zu, bevor der HBsAg-Test positiv wird, sowie möglicherweise in einer späteren Phase der Infektion, nachdem der Test wieder negativ geworden ist. Diese Lücken der Routinetestung lassen sich entweder durch zusätzliche Tests oder eine sogenannte Quarantänelagerung verringern. Bei HCV und HIV lässt sich das serologische Fenster durch eine empfindliche Untersuchung auf Virusgenom weitgehend, wenn auch nicht vollständig schließen. Beim HBV ist der Zeitgewinn vor dem positiven $\mathrm{HBsAg-Test} \mathrm{noch} \mathrm{stärker} \mathrm{als} \mathrm{bei}$ der HIV- und der HCV-Testung von der Empfindlichkeit der NAT abhängig, weil bereits auf einen Virusbestandteil getestet wird. Die mögliche späte Infektiosität bei wieder negativem HBsAg-Test wird fast vollständig durch einen zusätzlichen Antikörpertest, den Anti-Hepatitis-B-Core-Antigen (HBc)Test, erfasst. Anti-HBc erfasst allerdings generell bei Spendern eine zurückliegende Infektion mit HBV, wobei Blutprodukte der Spender in den allermeisten Fällen nicht mehr infektiös sind.

Bei der Quarantänelagerung wird vor der Freigabe der Gewebe der Lebendspender nach einem angemessenen Zeitraum noch einmal getestet, so dass eine frische Infektion durch einen bei der Zweittestung positiven Anti-HCV-, AntiHIV-, HBsAg- oder Anti-HBc-Test erkannt würde. Bei Leichenspendern wird, soweit möglich, der Empfänger eines Organs nach einer angemessenen Frist auf HIV-, HBV- und HCV-Parameter nachuntersucht, bevor die Gewebe zur Transplantation freigegeben werden. Sowohl zusätzliche Testung als auch Quarantäne führen zu einer aufwendigeren Logistik, möglicher Aussonderung von knappen Gewebetransplantaten und hohen zusätzlichen Kosten, die dem Sicherheitsgewinn gegenübergestellt werden müssen.

Als dritte Sicherheitsmaßnahme gibt es für einige Gewebe die Möglichkeit einer Pathogeninaktivierung.

In der einschlägigen Europäischen Richtlinie 2006/17/EG (als eine Durchführungsrichtlinie der dem Gewebegesetz zugrunde liegenden Richtlinie 2004/23/EG) werden für die Testung von Gewebespendern nur die Standardtestung mittels Anti$\mathrm{HCV}$, Anti-HIV und HBsAg sowie ein Anti-HBc-Test gefordert. In Deutschland existieren zwei geweberelevante Richtlinien der BÄK, eine zum «Führen einer Knochenbank» [1] und eine zum «Führen einer (Augen-)Hornhautbank» [2], die strengere Anforderungen, insbesondere in Bezug auf die NAT-Testung, stellen.

In der vorliegenden Arbeit wird versucht, anhand der gewebespezifischen Spendercharakterisierung, Untersuchungsmethoden sowie Herstellungs- und Inaktivierungsverfahren eine Bewertung der Virus-NAT bei Gewebespendern vorzunehmen, diese dem aktuellen Stand der medizinischen Wissenschaft auf dem Gebiet der Virusdiagnostik gegenüberzustellen und Algorithmen zur Gewährleistung der Virussicherheit zu entwickeln.

\section{Erfahrungen aus dem Blutspendewesen}

Bis zum Beginn der 1980er-Jahre war mit der Gabe von überlebenswichtigen Blutprodukten und Plasmaderivaten unmittelbar das Risiko einer Virushepatitis verbunden. Mit dem Aufkommen von HIV und dem erworbenen Immundefektsyndrom (AIDS) wurden neue Anstrengungen zur Vermeidung von Infektionen unternommen. Viele Plasmapools aus oft mehreren zehntausend Plasmen enthielten zu Beginn der HIV-Epidemie infektiöse Spenden und es kam zu Übertragungen durch die nicht ausreichend inaktivierten Endprodukte. Daher wurden Verfahren zur zuverlässig validierten Virusinaktivierung der daraus hergestellten Produkte entwickelt. Um den Sicherheitsspielraum dieser Verfahren zu erhöhen, wurde bereits die Viruslast im Ausgangsplasmapool weitestgehend begrenzt. Es wurden Verfahren eingesetzt, die das Virus oder seine Bestandteile mit sehr hoher Empfindlichkeit erfassten, zum Beispiel mit NAT wie der PolymeraseKettenreaktion (PCR). Aus Kostengründen und weil aufgrund der nachgeschalteten Virusinaktivierung nicht unbedingt jede niedrigtitrige Spende erfasst werden musste, wurden für das Screening mittels NAT-Testung zunächst mehrere 
Proben zusammengeführt (gepoolt) und in einem zweiten Schritt die für ein positives Ergebnis verantwortliche Spende identifiziert.

Blutspendedienste, die aus Vollblutspenden sowohl zelluläre Blutprodukte als auch Plasma zur Herstellung von Plasmaderivaten gewannen, sahen sich mit der Frage konfrontiert, ob die aus der Testung des Plasmas gewonnene Information der frischen Infektion nicht auch Auswirkungen auf die zellulären Produkte haben müsse. Es ging dabei nicht mehr darum, die Viruslast zu begrenzen, sondern möglichst jede serologisch nicht erfasste Infektion zu erkennen, was höhere Anforderungen an die Sensitivität der NAT-Tests und deren Durchführung stellte.

Da seronegativ getestete frische HCV-Infektionen des Spenders aus Rückverfolgungsuntersuchungen (nach Serokonversion des Spenders wurden in Deutschland durch Rückverfolgung 38 Infektionen der Empfänger von 1990 bis 1997 dokumentiert) als großes Infektionsproblem der Blutspende bekannt waren, führte das PEI 1998 die NAT auf HCV-Genome für zelluläre Blutprodukte verbindlich ein $[3,4]$. Dabei wurde die minimale Empfindlichkeit für die Einzelspende vorgegeben, die Möglichkeit des Pooling und die Festlegung der Poolgröße und Logistik wurde den Anwendern überlassen. Bei der $\mathrm{HCV}$-Infektion lässt sich die Zeit bis zum Nachweis von HCV im Plasma von Blutspendern durch einen zusätzlichen NAT um 41-60 Tage auf 15-22 Tage verkürzen [5].

Eine analoge Reduzierung des serologischen Fensters ist auch für den im Blutspendewesen am meisten gefürchteten Infektionserreger, das HIV, möglich. Untersuchungen der Interorganizational Task Force on Nucleic Acid Amplification Testing of Blood Donors in den USA zeigten eine Reduktion des diagnostischen Fensters für HIV durch Einführung der HIVNAT um 10-15 Tage auf zirka 10 Tage [5]. Obwohl die Häufigkeit von HIV-Infektionen durch Blutprodukte auch ohne NAT-Testung bereits auf weniger als 1:1 000000 geschätzt wurde (4 dokumentierte HIV-Übertragungen durch Blutprodukte von 1998 bis 2000), ordnete das PEI 2003 die Einführung der HIV-1-NAT für zelluläre Blutprodukte und gefrorenes Frischplasma an [6].

Bei HBV wird mit dem HBsAg-Test (im Gegensatz zur HCVund HIV-Antikörpertestung) ein Virusbestandteil nachgewiesen, der noch dazu gegenüber den kompletten HBV-Partikeln in hohem Überschuss gebildet wird. Dennoch besteht ein sehr wesentliches Restrisiko, da bei Erreichen der Nachweisgrenze der besten verfügbaren HBsAg-Tests im Mittel bereits 2000 (in einem seltenen Fall sogar 100 000) Viruspartikel/ml Plasma vorliegen. Verschärft wird das Problem dadurch, dass der Zeitraum der Verdoppelung der Viruslast im Plasma in der Frühphase viel länger als bei HCV oder HIV ist und daher ein infektiöser Spender mehrere Wochen HBsAg-negativ sein kann. Deswegen hängt die Verkürzung des serologischen Fensters bei HBV in sehr viel stärkerem Maße als bei HCV und HIV von der Empfindlichkeit der NAT ab. Ein weiterer Vorteil der HBV-NAT liegt in der Detektion von Virusmutatio- nen, die mit dem klassischen HBsAg-Test nicht nachweisbar sind. Eine Arbeit über die NAT-Ergebnisse der Blutspendedienste des Deutschen Roten Kreuzes (DRK) von 1997 bis 2005 an 31524571 Spenden zeigt, dass bei 23 dieser Spenden HCV-, bei 7 dieser Spenden HIV-1- und bei 43 dieser Spenden HBV-Infektionen mittels alleiniger NAT-Testung gefunden werden konnten [7]. Bei HBV wären 21/43 Spenden auch mit dem 2006 in Deutschland für alle Blutspender eingeführten Anti-HBc-Test gefunden worden. Die Anzahl der verbliebenen 22/43 nur HBV-NAT-positiven Spenden ist damit mit den 23 alleinig HCV-NAT-positiven Spenden vergleichbar. Insofern bringt die zusätzliche freiwillige NAT-Testung auf HBV für die Frühphase der Infektion einen ähnlich hohen Sicherheitsgewinn wie bei HCV. Inwieweit diese Daten auf andere Blutspendeorganisationen mit anders zusammengesetzten Spenderpopulationen übertragen werden können, ist allerdings nicht klar. Es gibt aber keine Anhaltspunkte, dass bei diesen Organisationen die HBV-NAT von geringerem Wert ist. Bei HBV besteht ein zusätzliches Infektionsproblem. Früher glaubte man, mit dem Verschwinden des HBsAg oder spätestens mit dem Erscheinen des Anti-HBs sei die HBV-Infektion ausgeheilt. Das erneute Aufflackern der Infektion bei Immunsuppression oder Transplantation zeigt aber, dass meistens die Infektion vom Immunsystem nur mehr oder weniger gut in Schach gehalten wird und das Virus weiter in der Leber, möglicherweise auch in anderen Organen, persistiert. In einem kleinen Teil dieser «okkult» infizierten HBV-Träger kann trotz nicht nachweisbarem HBsAg eine geringe Menge Virus im Blut vorliegen und zu Übertragungen führen. Die Phase nach dem Verschwinden des HBsAg, die im klassischen Sinn ausgeheilte Hepatitis B, wird in den meisten Fällen langfristig vom Anti-HBc-Test erfasst. Dieser Test erfasst neben den wenigen infektiösen auch alle aufgrund eines ausreichend hohen Anti-HBs-Titers nicht infektiösen Spender, was zwar zu einer hohen Sicherheit, aber auch zu einem hohen Verwurf bzw. Ausschluss von Spendern führt. In der Blutspende wurde deshalb die Möglichkeit eröffnet, den Spender trotz Nachweises von Anti-HBc wieder zur Spende zuzulassen, wenn er ein Anti-HBs von mindestens $100 \mathrm{IE} / \mathrm{l}$ hat und eine sehr empfindliche NAT auf HBV negativ ist [8]. Während nicht alle Autoren eine flächendeckende HBV-NAT-Testung in den USA empfehlen [5,9], ist sie in Japan vorgeschrieben. In Deutschland wird sie auf freiwilliger Basis für viele Blutspenden durchgeführt [7].

Die German Red Cross Study Group berechnete für ihre Organisation auf der Grundlage infektionsserologischer Befunde und HIV/HCV/HBV-NAT-Tests (Minipool) (31,5 Millionen Spenden, 1997-2005) das Risiko einer HIV-, HCV- bzw. HBV-Übertragung durch transfundierte Blutkomponenten (zelluläre Bestandteile oder Plasma) [7]. Danach liegt dieses für HIV bei 1:4,300 000, für HCV bei 1:10 880000 und für HBV bei 1:360 000. Andere Autoren kommen zu anderen Abschätzungen des Restrisikos in Abhängigkeit von der NAT-Testung $[10,11]$. 
In einer Untersuchung des PEI konnte vor kurzem belegt werden, dass auch die virale Kontamination von großen Plasmapools durch flächendeckende Einführung der HIV/HCV/ HBV-NAT deutlich reduziert werden konnte. Waren im Jahr 1996 17,8\% von 873 untersuchten Pools HCV-RNA-, 0,8\% HIV-1-RNA- und 0,5\% HBV-DNA-positiv, war im Jahr 2006 lediglich einer von 331 Pools HCV-RNA-positiv. Hier lag außerdem ein sehr niedriger Titer $(<10 \mathrm{IU} / \mathrm{ml})$ vor. HIV- und HBV-Genome waren nicht nachweisbar [12].

Trotz des unbestreitbaren Nutzens des NAT-Screenings müssen die Kosten der Einführung der Dreifach-PCR erwähnt werden, die pro Blutprodukt bei $>2$ EUR im 96-Pool und $>7$ EUR im 24-Pool liegen. Die Vorgehensweise des Pooling setzt ein umfangreiches Validierungsprogramm auf der Grundlage der durch das PEI vorgegebenen Empfindlichkeitsgrenzen in den Pools (HIV-1-RNA: $10000 \mathrm{IU} / \mathrm{ml}$; HCVRNA: $5000 \mathrm{IU} / \mathrm{ml}$, jeweils bezogen auf die Einzelblutspende) sowie der zutreffenden Committee for Proprietary Medicinal Products (CPMP)-Leitfäden CPMP/ICH/281/95 und CPMP/ ICH/381/95 voraus. Weitere Details zur Validierung der NATTechniken finden sich in einer PEI-Bekanntmachung aus dem Jahr 2000 [13]. In vielen Blutspendediensten Deutschlands sowie in vielen anderen Ländern hat sich die Untersuchung der Spenden in sogenannten Minipools (z.B. 10, 24, 48 oder 96 Proben/Pool) bewährt [10].

Wesentliche Grundlage der Vermeidung des Risikos von Virusübertragung sind auch weiterhin die Durchführung einer Spenderanamnese, der Ausschluss von Risikopersonen sowie klinische und infektionsserologische Untersuchungen. Schließlich ist für gefrorenes Frischplasma zur Transfusion auch weiterhin eine Quarantänelagerung von 4 Monaten durch das PEI vorgeschrieben.

Zusammengefasst lassen sich hinsichtlich der Virus-NAT folgende Schlussfolgerungen aus den Erfahrungen des deutschen Blutspendewesens ziehen:

- Die Einführung des Nachweises von HIV- und HCVGenom mittels NAT reduziert die diagnostische Lücke wesentlich.

- Spenderproben können für die NAT-Testung gepoolt werden, wenn das Verfahren des Pooling sowie die NAT-Tests in ihrer Sensitivität validiert wurden, weil die Infektionsphase mit einer Viruskonzentration unterhalb der Empfindlichkeitsgrenze bei HIV und HCV sehr kurz ist.

- Die HBV-NAT erhöht die Sicherheit von Blutprodukten, auch wenn eine serologische Untersuchung auf HBsAg und Anti-HBc erfolgt. Jedoch hängt die Effizienz der NAT für HBV sehr von der Nachweisgrenze ab.

- Die aktuellen Risiken der Übertragung von HIV oder HCV durch Blutprodukte sind mit 1:4 300000 bzw. 1:10 880000 extrem gering und für HBV mit 1:360 000 zwar deutlich höher, aber immer noch sehr gering.

Versucht man nunmehr, diese Erfahrungen auf das Gebiet der Gewebespende zu übertragen, stellen sich diverse Besonderheiten dar.

\section{Besonderheiten der Gewebespende}

\section{Gewebespender}

Der «klassische» Lebendspender ist nur in wenigen Bereichen des Tissue Banking anzutreffen. Exemplarisch sei auf die Spende von Femurköpfen nach Totalendoprothese des Hüftgelenkes sowie auf die Amnionspende hingewiesen. Jährlich werden in Deutschland zirka 20000 allogene Femurköpfe in orthopädischen/unfallchirurgischen Kliniken und vermutlich zirka 350 Amnien in Verbrennungskliniken bzw. ophthalmologischen Bereichen transplantiert. Femurkopf-Spender sind Patienten, nicht selten in höherem Alter und aus transfusionsmedizinischer Sicht in der Regel «Erstspender», die primär nicht beabsichtigen zu spenden, sondern erst wenige Tage vor der Operation über die Möglichkeit der Gewebespende informiert werden. Bei der Amnionspende werden die Mütter erst kurz vor der Geburt des Kindes über die Möglichkeit der Spende informiert. Damit besitzt dieses unselektierte Spenderklientel möglicherweise ein höheres Durchseuchungsrisiko als erstmalig Blutspendewillige bzw. Erstspender [14, 15], ist jedoch hinsichtlich der Virus-Risikofaktoren durch den aufklärenden Arzt genauso wie der Blutspendewillige zu befragen und kann entsprechend untersucht werden. Ein Poolen der Spenderblutproben erzeugt, wenn keine benachbarte Blutbank vorhanden ist, erhebliche logistische Probleme. Des Weiteren können für die Erstellung eines 24er-Pools in Abhängigkeit von den intraoperativ gewonnenen Geweben mindestens 4 Wochen vergehen. Hier wäre eine Zusammenarbeit mit einem Blutspendedienst anzustreben oder eine Einzeltestung vorzunehmen, was angesichts des höheren Preises der Gewebe vertretbar erscheint. Schließlich ist darauf hinzuweisen, dass die sogenannte Spender-Empfänger-Ratio (auf wie viele verschiedene Empfänger die Gewebetransplantate eines Spenders übertragen werden können) im Normalfall 1:1 beträgt.

Gewebe von verstorbenen Spendern, sogenannten Leichenspendern, wird in mehreren klinischen Fachgebieten transplantiert. Das Spektrum reicht von muskuloskelettalen Geweben (Knochen, Knorpel, Sehnen, Bänder, Faszien) über Augenhornhaut (Cornea) bis hin zu kardiovaskulären Allografts (Aorten- und Pulmonalklappe, Perikard, Venen, Arterien). Pro Jahr werden in Deutschland etwa 17 000-20 000 solcher Gewebe transplantiert. Bei diesen Spendern ist die Erfassung der Anamnese naturgemäß problematisch, da sie sich ausschließlich auf Angaben der Angehörigen (die meist für solche Fragen zu diesem Zeitpunkt nicht gut ansprechbar sind) bzw. der behandelnden Ärzte beschränken muss. Bei Multitransfundierten sind die serologischen und NAT-Ergebnisse wegen der eingetretenen Verdünnung von begrenzter Aussagekraft. Untersuchungen von Zou et al. [15] zeigen auf, dass Gewebespender hinsichtlich der HIV/HCV/HBV-Raten zwar eine geringere Durchseuchung hatten als die Normalpopulation, jedoch eine höhere als Erstblutspender. Zu ähnlichen Ergebnissen gelangen Yao et al. [16] in einer Untersuchung an 
12415 Spendern von muskuloskelettalem Gewebe in Australien. Die Spender-Empfänger-Ratio ist bei Augenhornhäuten 1:2, bei kardiovaskulären Transplantaten 1:1 bis 1:10 und bei muskuloskelettalen Geweben bis zu 1:40.

Prämortale Blutproben des Spenders liegen meistens nicht vor, insbesondere dann, wenn es sich nicht um Multiorganspender handelt (z.B. Spender aus rechtsmedizinischen Instituten) und Blutproben vom stationären Aufenthalt nicht mehr zur Verfügung stehen bzw. zu alt sind. Diese Proben sollten nicht älter als 7 Tage sein und bei +2 bis $+8{ }^{\circ} \mathrm{C}$ gelagert worden sein. Die Serum- bzw. Plasmatrennung muss nach der Abnahme erfolgt sein. Stehen diese prämortalen Proben nicht zur Verfügung, muss auf postmortales Blut zurückgegriffen werden, dessen Entnahme nur bis zu 24 h post mortem erfolgen kann (Transplantationsgesetz-Gewebeverordnung (TPGGewV)). Es könnte überlegt werden, dass die verantwortliche Person der Gewebeeinrichtung ( $\$ 20 \mathrm{c}$ des Arzneimittelgesetzes (AMG)) Abweichungen von diesen präanalytischen Vorgaben im Rahmen einer Gesamtrisikobewertung (z.B. dringende Indikation für eine Cornea bei nur geringfügiger zeitlicher Abweichung der prämortalen Probengewinnung von den Vorgaben) akzeptieren kann. Serologische Testverfahren weisen erfahrungsgemäß mit zunehmender Dauer der Postmortem-Blutentnahme höhere Unspezifitäten auf. Hier könnte eine entsprechend validierte und sensitive $\mathrm{HIV} / \mathrm{HBV} / \mathrm{HCV}$ NAT aus der Einzelprobe mit interner Kontrolle gegebenenfalls ausreichend aussagefähig sein, jedoch sind diesbezügliche Untersuchungen abzuwarten. Schließlich ist bei postmortalen Entnahmen nach $24 \mathrm{~h}$ die bakterielle Kontaminationsgefahr hoch und im Besonderen abzuwägen. Zudem ist in der Leiche von einem Abbau der Virusnukleinsäure auszugehen.

Viele der in Europa verfügbaren Testkits für die Infektionsserologie und Virus-NAT sind ohnehin nicht für post mortem gewonnene Proben validiert. Speziell Hämolyseeffekte können die NAT, aber auch serologische Tests beeinflussen [17, 18]. Für die Untersuchung postmortal gewonnener Proben sind nur wenige NAT-Tests validiert und Conformité Européenne (CE)-gekennzeichnet, wie z.B. die AmpliScreen Testfamilie von Roche. Hier ist ein verändertes Aufarbeitungsprotokoll zu befolgen, um eine mögliche Inhibition der Amplifikation zu vermeiden. Oft ist das Spenderblut durch Multitransfusionen verdünnt. All diese Umstände müssen in die Gesamtrisikoanalyse vor Freigabe der Gewebe einbezogen werden. Möglichkeiten der standardisierten Testung von postmortalem Blut werden jedoch zunehmend entwickelt [19]. Es erscheint sinnvoll, nicht nur die NAT, sondern die gesamte infektionsserologische Testung von Gewebespendern in Kooperation mit einer transfusionsmedizinischen Einrichtung durchzuführen, die einen eigenen Blutspendedienst und somit das gleiche Laboruntersuchungsspektrum vorhält. Einerseits besteht in dieser Kooperation eine sehr gute Logistik und Fachkompetenz, die den gesetzlichen Anforderungen des AMG, des TPG bzw. der Arzneimittel- und Wirkstoffherstellungsverordnung (AMWHV) entsprechen, andererseits können die Kosten bei Integration der Gewebespendertests in die Tagesroutine eines Blutspendedienstes niedrig gehalten werden.

Es bleibt festzuhalten, dass

- Gewebespender in der Regel unselektierte Erstspender sind, die im Fall der Lebendspende erst unmittelbar perioperativ, im Fall der Leichenspende (außer bei vorhandenem Organ-/Gewebespende-Pass) in der Regel nicht mit der Spendemöglichkeit konfrontiert werden und damit ein möglicherweise höheres Infektionsübertragungsrisiko im Vergleich zu erstmalig Blutspendewilligen aufweisen,

- die Anamneseerhebung bei Leichenspendern nur durch Befragung von behandelnden Ärzten sowie Angehörigen, Partnern und Freunden erfolgt und meist nicht alle Risikofaktoren erfassen kann,

- bei Leichenspendern meistens nur postmortale Blutproben gewonnen werden, deren diagnostischer Wert eingeschränkt sein kann,

- die Kooperation der Gewebebank mit einem Blutspendedienst auf dem Gebiet der Labortestung der Spender die effizienteste und kostengünstigste Lösung sein dürfte,

- die Spender-Empfänger-Ratio bei Leichenspendern bis zu 1:40 betragen kann, bei Lebendspendern jedoch zumeist nur 1:1 ist.

\section{Virusübertragungen durch Gewebetransplantate}

Bisher wurden nur sehr wenige Übertragungen durch klinisch relevante Viren beschrieben. Eine Zusammenstellung der bisher beschriebenen Übertragungen für muskuloskelettale Gewebe zeigt Tabelle 1.

Bemerkenswert ist die Beobachtung, dass die berichteten Virusübertragungen bis auf eine Ausnahme (gewaschene BoneTendon-Bone-Transplantate) durch nicht prozessierte bzw. nicht inaktivierte Gewebe erfolgten. Bei Empfängern von Geweben desselben infektiösen Spenders, die prozessiert (mechanische Präparationen, Spülungen zur Reinigung und Entfernung der Restblutmenge) und abschließend lyophilisiert worden sind, wurden keine Infektionen festgestellt. Für die Infektionsübertragung durch unprozessierte bzw. nicht inaktivierte Gewebe spricht auch ein Bericht von Marthy und Richter, die HIV in gefrorenen Rippentransplantaten, die bei einem HIV-infizierten Patienten zu Studienzwecken 44 h postmortal entnommen worden waren, nachweisen konnten [30]. Auch humanes HIV-2 blieb trotz Einfrier-/Auftauprozeduren in allogenem Knochengewebe nachweisbar [31].

Die Übertragung von nicht viralen Erregern durch allogene muskuloskeletale Gewebe ist ebenfalls beschrieben worden. Untersuchungen der Centers for Disease Control and Prevention (CDC) in US-amerikanischen Gewebebanken zeigten, dass im Zeitraum 2000-2002 (ca. 2 Millionen muskuloskelettale Transplantationen) insgesamt 26 Patienten transplantatbedingte Infektionen erlitten. 13/26 Patienten wurden durch allogene Sehnen (8), Femurkondylen (2), Spongiosa (2) und 
Tab. 1. HIV-, $\mathrm{HCV}$ - und HBVÜbertragungen durch muskuloskelettale Gewebe und Haut

\begin{tabular}{|c|c|c|c|}
\hline Virus & Jahr & Gewebe, das zur Übertragung führte & Autor \\
\hline HIV & 1984 & $\begin{array}{l}4 \text { Knochentransplantate; gefrierkonserviert, nicht sterilisiert, keine } \\
\text { HIV-Testung; Anm.: } 8 \text { Transplantate desselben Spenders ohne Infektion } \\
\text { der Empfänger }\end{array}$ & Schratt [20] \\
\hline HIV & 1984 & 1 Knochentransplantat; gefrierkonserviert, nicht sterilisiert, keine HIV-Testung & $\mathrm{CDC}[21]$ \\
\hline HIV & 1985 & $\begin{array}{l}3 \text { Knochentransplantate; gefrierkonserviert, nicht sterilisiert; Anm.: } 25 \text { Transplantate } \\
\text { desselben Spenders (Sehnen, Bänder, Knochen) nach Ethanol und/oder } \\
\text { Lyophilisation ohne Infektion der Empfänger }\end{array}$ & Simonds [22] \\
\hline HIV & 1987 & $\begin{array}{l}1 \text { Hauttransplantat; gefrierkonserviert, nicht sterilisiert, transplantiert bevor } \\
\text { (positives) HIV-Test-Ergebnis vorlag }\end{array}$ & Clarke [23] \\
\hline HIV & 1996 & 1 Femurkopf-Transplantat; gefrierkonserviert, nicht sterilisiert, keine HIV-Testung & $\operatorname{Li}[24]$ \\
\hline $\mathrm{HCV}$ & 1985 & 1 Femurkopf-Transplantat; gefrierkonserviert, nicht sterilisiert, keine HCV-Testung & Eggen [26] \\
\hline $\mathrm{HCV}$ & $1986-1990$ & $\begin{array}{l}2 \text { Knochentransplantate; gefrierkonserviert, nicht sterilisiert, keine HCV-Testung; } \\
\text { Anm.: retrospektive Untersuchung, beide Empfänger polytransfundiert, drei } \\
\text { Knochentransplantate desselben Spenders führten nicht zur Infektion }\end{array}$ & Pereira [27] \\
\hline $\mathrm{HCV}$ & 1991 & $\begin{array}{l}1 \text { Knochentransplantat, } 2 \text { Bone-Tendon-Transplantate, gefrierkonserviert, } \\
\text { nicht sterilisiert, Anti-HCV-Test (first generation) negativ }\end{array}$ & Conrad [25] \\
\hline $\mathrm{HCV}$ & 2000 & $\begin{array}{l}3 \text { Bone-Tendon-Bone-Transplantate; gefrierkonserviert, gewaschen, anti-HCV- } \\
\text { negativ, keine HCV-NAT; } 1 \text { Tibialis anterior-Sehne; kryokonserviert, anti-HCV } \\
\text { negativ, keine HCV-NAT; Anm.: } 16 \text { Knochentransplantate desselben Spenders } \\
\text { nach Prozessierung und Bestrahlung (16,4-19,7 kGy) ohne Infektion der Empfänger }\end{array}$ & Tugwell [28] \\
\hline $\mathrm{HBV}$ & 1954 & 1 Knochentransplantat; gefrierkonserviert, nicht sterilisiert, keine HBV-Testung & Shutkin [29] \\
\hline Virus & Jahr & Gewebe, das zur Übertragung führte & Autor \\
\hline HBV & 1984 & $\begin{array}{l}2 \text { Cornea-Transplantate; keine HBV-Testung, HBsAg-Nachweis aus Rückstellprobe } \\
\text { des Spenders } 9 \text { Monate nach Infektion, HBV-Status der Empfänger bei } \\
\text { Transplantation nicht bekannt }\end{array}$ & Hoft [33] \\
\hline $\mathrm{HBV}$ & 1990 & 1 Aortenklappen-Allograft; kryokonserviert, Spender war bekannt positiv für HBsAg & Morris [34] \\
\hline $\mathrm{HCV}$ & 2000 & $\begin{array}{l}1 \text { Vena-saphena-Transplantat; kryokonserviert, gewaschen, Antibiotika, Anti-HCV- } \\
\text { negativ, keine HCV-NAT }\end{array}$ & Tugwell [28] \\
\hline
\end{tabular}

Tab. 2. HCV- und HBV-Übertragungen durch Cornea und kardiovaskuläre Gewebe
Menisci (1) mit Clostridium septicum bzw. Clostridium sordelli infiziert. Alle Gewebe waren zwar aseptisch entnommen, jedoch keinem terminalen Desinfektions- bzw. Sterilisationsverfahren unterzogen worden. Weitere 11/26 Patienten waren mit Gram-negativen Bakterien infiziert worden, in zwei Fällen konnte der Erreger nicht eindeutig ermittelt werden. Die übertragenen Transplantate waren zumeist frisch oder gefrierkonserviert, in einem Fall auch gefriergetrocknet [32]. Folglich sind bei muskuloskelettalen Geweben die Übertragungen von Bakterien, verglichen mit Viren, die größere Gefahr.

HIV-, HCV- bzw. HBV-Übertragungen durch Cornea bzw. kardiovaskuläre Gewebe sind Raritäten, was vor allem auf die Blut- und Lymphgefäßfreiheit sowie den bradytrophen Stoffwechsel der Zellen der Augenhornhaut bzw. das umfassende Prozessieren sowie die geringe Anzahl an Transplantationen bei den kardiovaskulären Geweben zurückzuführen sein dürfte. Eine Übersicht zeigt Tabelle 2.

Vermutlich aufgrund der sehr geringen oder fehlenden Viruslast, da physiologisch keine Blutzellen in die Cornea penetrieren können, hat die Übertragung von Augenhornhäuten HIVbzw. HCV-infizierter Spender nicht zur Infektion des Empfän- gers geführt [17, 21, 22, 27, 35-37]. Bis heute sind keine HIVbzw. HCV-Übertragungen durch Corneae beschrieben worden. In der Studie von Sengler et al. [38] waren bei $6 \mathrm{HBV}$ bzw. 6 HCV-NAT-positiven Spendern Virusgenome im Corneagewebe (zentral und corneoskleraler Ring) nicht nachweisbar. Lediglich im Kulturmedium konnten in zwei Fällen mittels hochsensitiver NAT niedrige HCV-Genommengen (50-100 Kopien/ml) gefunden werden. Im Gegensatz hierzu fanden Lee et al. [39] in 7 von 29 Corneae von HCV-seropositiven Spendern HCV-Genom. Dieser Befund spräche somit für die Durchführung der HCV-NAT als weitere Sicherheitsmaßnahme bei Corneaspendern. Glasser [40] berichtete 1998 im Rahmen einer Untersuchung der Eye Bank Association of America (EBAA), dass bei der Transplantation von mehr als 400000 ausschließlich serologisch getesteten Corneatransplantaten in einem 12-jährigen Beobachtungszeitraum in den USA keine HIV-, HBV- bzw. HCV-Infektionen nachgewiesen wurden. Die in Tabelle 2 aufgeführte HBV-Übertragung aus dem Jahr 1984 [33] ist plausibel, jedoch nicht schlüssig bewiesen. Klinisch relevante Virusübertragungen durch Augenhornhäute wurden vereinzelt für Herpes-simplex-Viren 
(HSV) berichtet [41, 42]. Problematisch ist die mehrfach beschriebene Übertragung des neurotropen Rabiesvirus durch Corneatransplantate [43] sowie die Möglichkeit der Übertragung pathologischer Prionen [44].

Die in Tabelle 2 beschriebene HBV-Übertragung durch ein Aortenklappen-Allograft muss ebenfalls mit Zurückhaltung betrachtet werden, da der Spender HBsAg-positiv war und das Allograft trotzdem transplantiert wurde. HIV- und HCVÜbertragungen durch Herzklappen sind nicht bekannt. Pereira et al. [27] beschreiben 1993 die Transplantation von 3 Herzklappen von 2 HCV-RNA-positiven Spendern, die nicht zur Infektion des Empfängers geführt haben. Die in Tabelle 2 beschriebene HCV-Übertragung durch ein Venentransplantat ist der einzige bisher beschriebene Fall einer Virusübertragung durch Gefäße. Kardiotrope Viren, wie z.B. Cytomegalievirus, Parvovirus B19, Enterovirus, Adenovirus oder Influenzavirus, werden weitaus häufiger detektiert und stellen derzeit einen Schwerpunkt von Untersuchungen zur Virusübertragung durch kardiovaskuläre Allografts dar [45].

Zusammengefasst bleibt festzuhalten, dass

- Gewebetransplantate HIV, HCV und HBV übertragen können, dies jedoch ein seltenes Ereignis ist,

- die Übertragung anderer Viren, z.B. HSV durch Cornea, möglicherweise ein größeres Problem darstellt,

- HIV-, HCV- bzw. HBV-Übertragungen bisher fast ausschließlich durch nicht prozessierte bzw. nicht virusinaktivierte, muskuloskelettale Gewebe beschrieben wurden,

- Übertragungen dieser Viren durch Augenhornhäute bzw. kardiovaskuläre Gewebe Raritäten sind,

- das Prozessieren von Geweben (mechanische Präparationen, Spülungen zur Entfernung der Restblutmenge, Lyophilisation) offenbar zu einer Reduktion des Infektionsrisikos führt,

- soweit die biologischen Eigenschaften des Gewebes im notwendigen Umfang erhalten werden, durch die Integration von Virusinaktivierungsverfahren in das Herstellungsverfahren weitere Sicherheit garantiert ist.

\section{Inaktivierungsverfahren}

Der Einsatz von validierten chemischen oder physikalischen Verfahren zur Virusinaktivierung (HIV, HBV, HCV bzw. geeignete Modellviren) ist in Abhängigkeit von den biologischen Eigenschaften und dem späteren therapeutischen Einsatz des Gewebes grundsätzlich anzustreben, solange dadurch die Eignung des Gewebes nicht wesentlich beeinträchtigt wird. Folgende Verfahren sind für avitale muskuloskelettale Gewebe bekannt: das Peressigsäure-Ethanol-Unterdruckverfahren, das Thermodesinfektionsverfahren (Marburger Knochenbank), die Gammastrahlen-Behandlung sowie Kombinationen aus Gammabestrahlung und chemischen Inaktivierungsschritten (Tutoplast-Prozess) [46].

Diese Verfahren müssen validiert werden. Sie führen in der Regel zu Abreicherungen klinisch relevanter Viren bzw. Mo- dellviren um mindestens $4 \log _{10}$-Stufen der tissue culture infectious dose ( TCID $\left._{50} / \mathrm{ml}\right)$. Damit sind sie in Kombination mit den anderen Sicherheitsmaßnahmen (Anamnese, Klinik, Serologie, NAT) geeignet, eine sehr hohe Virussicherheit der Gewebe zu gewährleisten. Knorpelgewebe, Menisci, Corneagewebe bzw. kardiovaskuläre Gewebe können diesen Verfahren nicht unterzogen werden. Abschließend sei erwähnt, dass auch die Gefriertrocknung zu einer gewissen Abreicherung (etwa 1-2 $\log _{10}$-Stufen) von viraler Infektiosität (z.B. beim vesikulären Stomatitis-Virus oder bei Enteroviren) führen kann [47]. Andere Viren werden jedoch durch die Gefriertrocknung kaum beeinträchtigt und die Infektiosität von lyophilisierten Viruspartikeln wird dauerhaft konserviert. Virusübertragungen durch lyophilisierte Plasmaprodukte, wie Faktor VIII und IX, sind bekannt. Die Lyophilisation ist generell kein für alle pathogenen Viren effektiver Schritt zur Virusreduktion, sie kann nur einen geringen additiven Effekt haben.

Zusammengefasst bleibt festzuhalten, dass

- eine Reihe von effizienten Virusinaktivierungsverfahren, vor allem für muskuloskelettale Gewebe, zur Verfügung steht,

- effiziente Inaktivierungsverfahren einen entscheidenden Beitrag zur Virussicherheit von Geweben darstellen und neben der Testung von Blut auf Virusmarker wenn immer möglich eingesetzt werden sollten,

- Inaktivierungsverfahren nur dann eingesetzt werden können, wenn die resultierende therapeutische Qualität der Gewebe akzeptabel ist; daher sind die derzeit bekannten Inaktivierungsverfahren für eine Reihe von Gewebezubereitungen (z.B. Augenhornhaut, kardiovaskuläre Gewebe, Menisci, Knorpelgewebe) nicht einsetzbar.

\section{Virus-NAT und weitere Maßnahmen der Risikoreduk- tion bei Gewebespendern}

Aus den bisher genannten Daten zur Virussicherheit von Geweben sowie in Anbetracht der Erfahrungen aus den Blutspendediensten werden folgende Prinzipien zur Reduktion des Risikos einer HIV-, HBV- bzw. HCV-Infektion bei Gewebespendern abgeleitet:

\section{Maßnahmen, die für alle Gewebespender zutreffen}

- Umfassende Anamnese zur Erfassung von Risikofaktoren für eine Virusinfektion (Festlegung der Details in Fachgesellschaften, BÄK und PEI auf der Grundlage bestehender Regelungen sowie der EU-Richtlinie 2006/17/EG).

- Klinische Untersuchung bzw. Inspektion des Spenders (Zeichen einer Infektionskrankheit, Zeichen der Zugehörigkeit zu einer HIV-, HCV- bzw. HBV-Risikogruppe).

- Labor-Basisprogramm: Anti-HIV-1/2, HBsAg, Anti-HBc, Anti-HCV, Treponema-pallidum-Hämagglutinationsassay (TPHA). 
- Es sollte unbedingt versucht werden, prämortale Proben zu erhalten und bis zum Spendezeitpunkt als Plasma/ Serum aufzubewahren.

- Sind Anti-HBc positiv und HBsAg negativ sowie in der anschließenden Untersuchung Anti-HBs $\geq 100$ IE/l und eine sensitive HBV-DNA-NAT aus der Einzelprobe negativ, kann die Spende in Bezug auf HBV verwendet werden.

\section{Zusätzliche Maßnahmen bei muskuloskelettalen Gewebespendern}

Bei Lebendspendern sind die HCV-, HIV- und HBV-NAT durchzuführen. Die Blutproben können analog zum im Blutspendewesen eingeführten Verfahren einem validierten Pooling unterzogen werden. Ein Entfallen der HCV-, HIV- und HBV-NAT-Testung kann allenfalls erwogen werden, wenn ein entsprechend validiertes Inaktivierungsverfahren eingesetzt wird. Bei diesem sollte auf der Grundlage des Europäischen Leitfadens CPMP/BWP/268/95 eine effektive Inaktivierung oder Entfernung von Viren nachgewiesen sein, die die vollständige Inaktivierung von Viren in der virämischen Phase gewährleistet. Hierzu sollte eine Risikobewertung erfolgen, die die Virusmenge im Ausgangsmaterial (Berücksichtigung der Blutmenge im Gewebe) sowie die Abreicherungskapazität des Verfahrens gewichtet. Die Kriterien für effektive Prozesse zur Virusabreicherung sind im Leitfaden CPMP/BWP/295/95 festgelegt und beinhalten unter anderem die Abreicherung von geeigneten behüllten und nicht behüllten Viren bzw. Modellviren um mindestens $4 \log _{10}$ TCID $50 /$ ml. Über die Akzeptanz des Verfahrens sowie den damit möglicherweise verbundenen Entfall der NAT-Testung entscheidet die zuständige Bundesoberbehörde, das PEI.

Bei Leichenspendern ist grundsätzlich eine NAT auf HCVRNA, HIV-RNA und HBV-DNA durchzuführen, die Einzeltestung kann dabei sinnvoll sein. Auf die Einschränkungen der NAT aus postmortalem Blut wird erneut hingewiesen. Validierte Inaktivierungsverfahren können analog zur Vorgehensweise bei Lebendspendern einen Verzicht der NAT-Testung begründen.

\section{Zusätzliche Maßnahmen bei Spendern von kardiovaskulärem Gewebe}

Kontaminiertes kardiovaskuläres Gewebe kann erwiesenermaßen HBV und HCV übertragen (Tab. 2), hat aber bei Negativität der serologischen Testung ein sehr geringes Restrisiko der HIV/HBV/HCV-Übertragung. Aufgrund der unbestrittenen Vorteile der NAT (Reduktion der diagnostischen Lücke und Ausschluss der sehr hohen Virämien), der Gewebemorphologie und der Spender-Empfänger-Ratio von bis zu 1:10 sollte die HIV/HCV/HBV-NAT-Testung jedoch als zusätzliche Sicherheitsmaßnahme erfolgen.

\section{Zusätzliche Maßnahmen bei Spendern von Augenhornhaut}

Die HCV-NAT soll durchgeführt werden. Die Testung auf Anti-HBc ist gemäß Richtlinie 2006/17/EG bzw. TPG-GewV generell vorgesehen. Jedoch gibt es schon in der Blutspende die Möglichkeit, Spender, die aufgrund zusätzlicher Ergebnisse vermutlich nicht infektiös sind, trotz positivem Anti-HBc zur Spende zuzulassen. In der Transfusionsmedizin, bei der mehr als $200 \mathrm{ml}$ Blut/Transfusionseinheit transfundiert werden, sind diese zusätzlichen Kriterien ein Anti-HBs $\geq 100$ IE/1 und eine negative HBV-Einzel-PCR. In Anbetracht der Tatsache, dass Corneae praktisch blut- und lymphfrei sind, sollte man bei positivem Anti-HBc-Test und negativer empfindlicher HBV-PCR die Möglichkeit eröffnen, die Cornea nach Einzelfallentscheidung zu transplantieren.

Um Spender im diagnostischen Fenster zu erkennen, wäre es denkbar, hier die derzeit in der BÄK-Richtlinie vorgeschriebene HCV-NAT auch durch die etwas weniger empfindlichen Tests auf HCV-Antigen zu ersetzen. Allerdings würden dann unterschiedliche Strategien bei Blut und bestimmten Geweben verfolgt. Zudem zeigen neuere Untersuchungen eine bei bestimmten HCV-Genotypen deutlich verringerte Sensitivität des Antigennachweises [48, 49].

\section{Quarantänelagerung}

Bei Spendern, die mittels NAT auf HCV-RNA, HIV-RNA und HBV-DNA negativ getestet wurden, kann auf eine Quarantänelagerung des entnommenen Gewebes verzichtet werden.

Dies entspricht den Vorgaben der EU-Richtlinie 2006/17/EG und führt zu einer deutlichen Verbesserung der Versorgungssituation mit kostbaren Gewebetransplantaten, da die Wiedereinbestellung von Lebendspendern bzw. die Nachtestung von Organempfängern erhebliche logistische Probleme hervorrufen, die nicht selten mit dem Verwurf des Gewebes verbunden sind. Zudem verhindert eine NAT des Spenders die Einlagerung potenziell infektiöser Gewebe und damit mögliche Kreuzkontaminationen bei der Lagerung.

Eine Quarantänelagerung kann ebenfalls entfallen, wenn die primär negativ getesteten Gewebe

- einer Prozessierung (validierte Entfernung der theoretisch zu einer Virusinfektion führenden Restblutmenge im Gewebe durch Präparationen bzw. Spülungen) unterzogen werden oder

- einem validierten und durch das PEI akzeptierten Inaktivierungsverfahren unterzogen werden oder

- einer begrenzten Haltbarkeit der Gewebezubereitung (unter 6 Wochen) unterliegen.

Werden die NAT-Tests nicht durchgeführt bzw. die Kriterien nicht erfüllt, so muss sich eine Quarantänelagerung gemäß den Vorgaben der für das Gewebe jeweils zutreffenden BÄKRichtlinien [1,2] anschließen. 
Tab. 3. Vorschlag zur virologischen Testung von Gewebespendern

\begin{tabular}{|c|c|c|c|c|}
\hline Gewebe & $\begin{array}{l}\text { Sero } \\
\operatorname{logie}^{\mathrm{a}}\end{array}$ & $\begin{array}{l}\mathrm{HCV}- \\
\mathrm{NAT}^{\mathrm{b}}\end{array}$ & $\begin{array}{l}\text { HIV- } \\
\text { NAT }^{b}\end{array}$ & $\begin{array}{l}\text { HBV- } \\
\text { NAT }^{b}\end{array}$ \\
\hline Muskuloskelettale Gewebe & $\mathrm{ja}^{\mathrm{d}}$ & $j a^{c}$ & $j a^{c}$ & $\mathrm{ja}^{\mathrm{c}}$ \\
\hline Kardiovaskuläre Gewebe & $\mathrm{ja}^{\mathrm{d}}$ & ja & ja & ja \\
\hline Cornea & $\mathrm{ja}^{\mathrm{e}}$ & ja & nein & nein \\
\hline
\end{tabular}

${ }^{a}$ Anti-HIV-1/2, Anti-HCV, HBsAg, Anti-HBc, TPHA.

${ }^{b}$ Eine Spezifizierung der Testung als Einzelprobe oder im Minipool wird nicht gefordert. Die erforderliche Nachweisgrenze der Testung sollte aber grundsätzlich, in Analogie zur Verfahrensweise in Blutspendediensten, auf die Einzelspende bezogen werden.

${ }^{\mathrm{c}}$ Bei validierten und durch das PEI akzeptierten Verfahren zur Virusinaktivierung kann die NAT entfallen.

${ }^{\mathrm{d}}$ Ist Anti-HBc reaktiv, Anti-HBs $\geq 100$ IE/l und HBV-DNA-NAT negativ, kann das Gewebe verwendet werden.

' Ist Anti-HBc reaktiv und HBV-DNA-NAT negativ, kann das Gewebe verwendet werden.

\section{Autologe Spender}

Bei Verwendung von autologen Geweben, z.B. im Rahmen des Tissue Engineering (autologe Knorpelzellkulturen, Keratinozytenkulturen usw.), kann auf Virus-NAT-Untersuchungen des Spenders verzichtet werden. Um die Häufigkeit der Kontaminationen von Kulturen weiterer Spender zu vermeiden, sollte jedoch, wie bei der autologen Blutspende eingeführt, die infektionsserologische Testung durchgeführt werden.

\section{Fazit}

1) Allogene Gewebetransplantate werden in der Regel elektiv transplantiert. Damit entfällt der lebensrettende Anspruch, der einen Verzicht auf maximale Sicherheitsmaßnahmen rechtfertigen könnte.

2) Die Vorschläge berücksichtigen die bisher bekannten epidemiologischen Daten zur Virusübertragung durch Gewebe, die Besonderheiten der einzelnen Spender, der Herstellungs- bzw. Inaktivierungsverfahren sowie der Logistik- und Kostenfragen.

3) Muskuloskelettale Gewebe besitzen infolge des zumeist hohen Blutgehalts der Gewebe, des umfangreichen Entnahmespektrums, der bisher beschrieben Übertragungen, der unterschiedlichen Herstellungsverfahren sowie der hohen Spender-Empfänger-Ratio ein signifikantes Risiko, HIV/HBV/HCV zu übertragen. Der Sicherheitsgewinn einer zusätzlich zu den vorgeschriebenen serologischen Tests auf HBsAg und Anti-HBc durchgeführten HBV-NAT ist nach neuesten Erkenntnissen [7, 50] relevant. Daher sollte bei muskuloskelettalen Gewebespendern eine HIV/HCV/HBV-NAT durchgeführt werden, wobei die Einschränkungen der NAT aus postmortalem Blut berücksichtigt werden müssen (Tab. 3).
4) Eine Durchführung der NAT-Testung als Einzelprobe oder im Minipool wird nicht grundsätzlich gefordert. Die erforderliche Nachweisgrenze der Testung sollte aber grundsätzlich, in Analogie zur Verfahrensweise in Blutspendediensten, auf die Einzelspende bezogen werden.

5) Effektive Virusinaktivierungsverfahren sollten, wenn immer möglich, angewendet werden und können den Verzicht auf die NAT-Testung rechtfertigen, wenn durch eine Kombination von Verfahren eine vollständige Inaktivierung von Viren aus der hochvirämischen Phase gewährleistet ist.

6) Nicht getestetes, kontaminiertes kardiovaskuläres Gewebe kann HBV und HCV übertragen, hat aber bei Durchführung der serologischen Testung ein sehr geringes Restrisiko der HIV/HBV/HCV-Übertragung. Aufgrund der offenkundigen Vorteile der NAT (Reduktion der diagnostischen Lücke), des Erhalts der Gewebemorphologie ohne Inaktivierung und der Spender-EmpfängerRatio von bis zu 1:10 sollte die HCV-, HIV- und HBVNAT-Testung jedoch als zusätzliche Sicherheitsmaßnahme erfolgen (Tab. 3). Diese Empfehlung folgt auch den Vorgaben der österreichischen Homograft-Richtlinie [51].

7) Augenhornhäute besitzen aus physiologisch-morphologischer sowie epidemiologischer Sicht das geringste HIV/HBV/HCV-Übertragungsrisiko. In Anbetracht der deutlichen Reduktion der serologischen Latenzphase sowie dem beschriebenen HCV-Nachweis in Corneagewebe von HCV-infizierten Spendern [39] sollte die HCV-NAT durchgeführt werden. Der Test auf Anti$\mathrm{HBc}$ ist gesetzlich vorgeschrieben, jedoch müssen in Analogie zur Blutspende die Kriterien definiert werden, bei denen Corneae trotz positivem Anti-HBc-Test transplantiert werden können (Tab. 3). Dies könnte bei negativer empfindlicher HBV-NAT eine Einzelfreigabe unter besonderer Beachtung eventueller anamnestischer Risiken sein.

8) Eine Quarantänelagerung der Gewebe eines Spenders kann bei initialer Durchführung und Negativität der HIV/HCV/HBV-NAT und/oder anderen Kriterien (Restblutmenge, Virusinaktivierung, begrenzte Haltbarkeit) entfallen.

9) Aufgrund der vielfältigen Synergieeffekte mit der Transfusionsmedizin sollten Gewebebanken die Testung der infektionsserologischen bzw. molekularbiologischen Laborparameter gemeinsam mit Blutspendediensten durchführen.

10) Gewebebanken sollten versuchen, über abgestimmte Spenderprogramme Synergieeffekte bei der Gewinnung des Gewebes zu erzielen. Die für einen Spender in einem zugelassenen Labor ( $\$ 20$ b AMG) erhobenen Befunde wären dann für alle beteiligten Gewebebanken verwendbar, was aufgrund der Vermeidung von Mehrfachtestungen zu einer Kostenreduktion führen würde. 


\section{Literatur}

1 Bundesärztekammer: Richtlinien zum Führen einer Knochenbank. Dtsch Arztebl 2001;98:A1011-1016.

2 Bundesärztekammer: Richtlinien zum Führen einer Hornhautbank. Dtsch Arztebl 2000;97:A2122-2124.

3 Paul-Ehrlich-Institut: Bekanntmachung über die Ergebnisse des Stufenplanverfahrens zur Verminderung des Risikos von Hepatitis B-, Hepatitis Cund HIV-Infektionen bei Empfängern von Erythrozytenkonzentraten vom 25,02 1998. Bundesanzeiger 1998;63:4477.

4 Paul-Ehrlich-Institut: Abwehr von Arzneimittelrisiken: Verminderung des Risikos von Hepatitis CVirus-Kontaminationen in Thrombozytenkonzentraten vom 05,06 1998. Bundesanzeiger 1998;114 8775

$\checkmark 5$ Busch MP, Kleinman SH, Jackson B, Stramer SL, Hewlett I, Preston S: Committee report. Nucleic acid amplification testing of blood donors for transfusion-transmitted infectious diseases: Report of the Interorganizational Task Force on Nucleic Acid Amplification Testing of Blood Donors. Transfusion 2000;40:143-159.

6 Paul-Ehrlich Institut: Verminderung des Risikos von HIV-1-Infektionen durch zelluläre Blutprodukte und gefrorenes Frischplasma - Anordnung der Testung auf HIV-1-RNA mit NAT. Bundesanzeiger 2003;103:12269.

7 Hourfar MK, Jork, C, Schottstedt V, Weber-Schehl M, Brixner V, Busch MP, Geusendam G, Gubbe K, Mahnhardt C, Mayr-Wohlfart U, Pichl L, Roth WK, Schmidt M, Seifried E, Wright DJ: Experience of German Red Cross blood donor services with nucleic acid testing: results of screening more than 30 million blood donations for human immunodeficiency virus-1, hepatitis C virus, and hepatitis B virus. Transfusion 2008:48:1541-1544.

8 Paul-Ehrlich-Institut: Abwehr von Arzneimittelrisiken; Testung auf Antikörper gegen Hepatitis-BCore-Antigen (anti-HBc) im Blutspendewesen. Bundesanzeiger 2006; 109:4370.

$\checkmark 9$ Busch MP: Should HBV DNA NAT replace HBsAg and/or anti-HBc screening of blood donors? Transfus Clin Biol 2004;11:26-32.

10 Stramer SL, Glynn SA, Kleinman SH, Strong DM, Caglioti S, Wright DJ, Dodd RY, Busch MP: Detection of HIV-1 and HCV infections among antibody-negative blood donors by nucleic acid-amplification testing. N Eng J Med 2004;351:760-768.

-11 Offergeld R, Ritter S, Hamouda O: HIV, HCV, $\mathrm{HBV}$ and syphilis infections among blood donors in Germany 2005. Bundesgesundheitsbl Gesundheitforsch Gesundheitsschutz 2007;50:1221-1231.

12 Nübling CM, Unkelbach U, Chudy M, Seitz R: Effect of viral nucleic acid testing on contamination frequency of manufacturing plasma pools. Transfusion 2008;48:822-826.

13 Paul-Ehrlich-Institut: Anforderungen an die diagnostische Erprobung von NAT zum Nachweis, zur Bestätigung und zur quantitativen Bestimmung von Markern von HIV-Infektionen sowie von Hepatitis $\mathrm{B}$ und $\mathrm{C}$ in Proben menschlichen Ursprungs. Bundesanzeiger 2000;213:21805.

14 Challine D, Pellegrin B, Bouvier-Alias M, Rigot P, Laperche L, Pawlotsky JM: HIV and hepatitis C virus RNA in seronegative organ and tissue donors. Lancet 2004:364:1611-1612.

-15 Zou S, Dodd RY, Stramer SL, Strong DM; Tissue Safety Study Group: Probability of viremia with HBV, HCV, HIV, and HTLV among tissue donors in the United States. N Engl J Med 2004;351: 751-759.

-16 Yao F, Seed C, Farrugia A, Morgan D, Cordner S, Wood D, Zheng MH: The risk of HIV, HBV, HCV and HTLV infection among musculoskeletal tissue donors in Australia. Am J Transplant 2007;7:27232726.
Challine D, Roudot-Thoraval F, Sabatier P, Dubernet F, Larderie P, Rigot P, Pawlotsky JM: Serological viral testing of cadaveric cornea donors. Transplantation 2006;82:788-793.

18 Heim A, Wagner D, Rothämel T, Hartmann U, Flik J, Verhagen W: Evaluation of serological screening of cadaveric sera for donor selection for cornea transplantation. J Med Virol 1999;58:291-295.

19 Strong DM, Nelson K, Pierce M, Stramer SL: Preventing disease transmission by deceased tissue donors by testing blood for viral nucleic acid. Cell Tiss Bank 2005;6:255-262.

20 Schratt HE, Regel G, Kiesewetter B, Tscherne H: HIV-Infektion durch kältekonservierte Knochentransplantate. Unfallchirurg 1996;9:679-684.

21 CDC, Centers for Disease Control and Prevention: Transmission of HIV through bone transplantation: case report and public health recommendations. MMWR 1988;37:597-599.

22 Simonds RJ, Holmberg SD, Hurwitz RL, Coleman TR, Bottenfield S, Conley LJ, Kohlenberg SH, Castro KG, Dahan BA, Schable CA: Transmission of human immunodeficiency virus type 1 from a seronegative organ and tissue donor. N Engl J Med 1992;326:726-732.

23 Clarke JA: HIV transmission and skin grafts. Lancet 1987;i:983.

24 Li CM, Ho YR, Liu YC: Transmission of human immunodeficiency virus through bone transplantation: A case report. J Formosan Med Assoc 2001; 100:350-351.

25 Conrad EU, Gretch DR, Obermeyer KR, Moogk MS, Sayers M, Wilson JJ, Strong DM: Transmission of the hepatitis-C virus by tissue transplantation. J Bone Joint Surg Am 1995;77:214-224.

26 Eggen BM, Nordbo SA: Transmission of HCV by organ transplantation. N Engl J Med 1992;326:411.

27 Pereira BJ, Milford EL, Kirkman RL, Levey AS, Tomford WW, Leibowitz H, Rhodes M, Quan S, Wilbur JC: Low risk of liver disease after tissue transplantation from donors with HCV. Lancet 1993;341:903-904.

28 Tugwell BD, Patel PR, Williams IT, Hedberg K, Chai F, Nainan OV, Thomas AR, Woll JE, Bell BP, Cieslak PR: Transmission of hepatitis $\mathrm{C}$ virus to several organ and tissue recipients from an antibody-negative donor. Ann Intern Med 2005;143: 648-654.

29 Shutkin NM: Homologous-serum hepatitis following the use of refrigerated bone-bank bone. J Bone Joint Surg 1954;36A:160-162.

30 Marthy S, Richter M: Human immunodeficiency virus activity in rib allografts. J Oral Maxillofac Surg 1998;56:474-476.

31 Cook SD, Salkeld SL, Prewett AB: Simian immunodeficiency virus (human HIV-II) transmission in allograft bone procedures. Spine 1995;20:13381342.

32 CDC, Centers for Disease Control and Prevention: Update: allograft-associated bacterial infections. MMWR 2002;51:207-210.

33 Hoft RH, Pflugfelder SC, Forster RK, Ullman S, Polack FM, Schiff ER: Clinical evidence for hepatitis B transmission resulting from corneal transplantation. Cornea 1997;16:132-137.

34 Morris A, Strickett MG, Barratt-Boyes BG: Use of aortic valve allografts from hepatitis B surface antigen-positive donors. Ann Thorac Surg 1990;49: 802-805

35 Schwarz A, Hoffman F, L'Age Stehr J, Tegzess AM, Offermann G: Human immunodeficiency virus transmission by organ donation: Outcome in cornea and kidney recipients. Transplantation 1987; 44:21-24.
6 Krajden M, Bishai F, Quan C, Mahony J, Brunton J, Rootman D, Zhao J, Lau W, Snell G, Maurer J, Kesten S, Colby D: Multi-organ donor transmission of hepatitis $\mathrm{C}$ virus to five solid organ transplant recipients and lack of transmission to corneal transplant recipients. Clin Diagn Virol 1995;3:113-121.

37 Caron MJ, Wilson R: Review of the risk of HIV infection through corneal transplantation in the United States. J Am Optom Assoc 1994;65:173-178.

38 Sengler V, Reinhard T, Adams O, Gerlich W, Sundmacher R: Testing of corneoscleral discs and their culture media of seropositive donors for hepatitis $\mathrm{B}$ and $\mathrm{C}$ virus genomes. Graefes Arch Clin Exper Ophthalmol 2001;239:783-787.

39 Lee HM, Naor J, Alhindi R, Chinfook T, Krajden M, Mazzulli T, Rootman DS: Detection of hepatitis $\mathrm{C}$ virus in the corneas of seropositive donors. Cornea 2001;20:37-40.

40 Glasser DB: Serologic testing of cornea donors. Cornea 1998;17:123-128.

41 Robert PY, Adenis JP, Pleyer U: How 'safe' is corneal transplantation? A contribution on the risk of HSV-transmission due to corneal transplantation. Klin Monatsbl Augenheilkd 2005;222:870-873.

42 Thuret G, Acquart S, Gain P, Thuret G, Dumollard JM, Manissolle C, Campos-Guyotat L, Gain P, Labetoulle M, Bourlet T: Ultrastructural demonstration of replicative herpes simplex virus type 1 transmission through corneal graft. Transplantation 2004;77:325-326.

43 Bronnert J, Wilde H, Tepsumethanon V, Lumlertdacha B, Hemachudha T: Organ transplantations and rabies transmission. J Travel Med 2007;14: 177-180.

44 Maddox RA, Belay ED, Curns AT, Zou WQ, Nowicki S, Lembach RG, Geschwind MD, Haman A, Shinozaki N, Nakamura Y, Borer MJ, Schonberger LB: Creutzfeldt-Jakob disease in recipients of corneal transplants. Cornea 2008;27:851-854.

45 Donoso Mantke O, Meyer R, Prösch S, Nitsche A, Leitmeyer K, Kallies R, Niedrig M: High prevalence of cardiotropic viruses in myocardial tissue from explanted hearts of heart transplant recipients and heart donors: a 3-year retrospective study from a German patients' pool. J Heart Lung Transplant 2005;24:1632-1638.

46 Pruss A, Katthagen BD: Musculoskeletal tissue banks: Legal foundations and graft safety. Orthopäde 2008;37:749-755.

47 Uhlenhaut C, Dörner T, Pauli G, Pruss A: Effects of lyophilization on the infectivity of enveloped and non-enveloped viruses in bone tissue. Biomaterials 2005;26:6558-6564.

48 Nübling CM, Unger G, Chudy M, Raia S, Löwer J: Sensitivity of HCV core antigen and RNA detection in the early infection phase. Transfusion 2002; 42:1037-1045.

49 Tuke PW, Grant PR, Waite J, Kitchen AD, Eglin RP, Tedder RS: Hepatitis C virus window-phase infections: closing the window on hepatitis $\mathrm{C}$ virus. Transfusion 2008;48:594-600.

50 Komiya Y, Katayama K, Yugi H, Mizui M, Matsukura H, Tomoguri T, Miyakawa Y, Tabuchi A, Tanaka J, Yoshizawa H: Minimum infectious dose of hepatitis B virus in chimpanzees and difference in the dynamics of viremia between genotype A and genotype C. Transfusion 2008;48:286-294.

51 Österreichisches Bundesinstitut für Gesundheitswesen (ÖBIG) und Bundesministerium für soziale Sicherheit und Generationen: Richtlinien für Homograft-Banken. ÖBIG, April 2002. 